\title{
Carillas de porcelana como solución estếlica en dientes anteriores: informe de doce casos
}



\author{
Porcelain veneers as aeshthetic solution for front reeth: report of twelve cases
}

\section{de Răbago-Vega, José* Tello-Rodríguez, Ana |sabel ${ }^{* *}$}

* Postgrado en Prótesis y Oclusión por el Instituto Pankey, Miami (USA). Práctica privada en prótesis e implantes en Madrid.

** Licenciada en Odontología por la UCM.
Resumen: La estética es hoy en día una parte importante de las relaciones sociales y profesionales. Los sistemas restauradores cerámicos sin núcleo metálico nos han permitido avanzar en los resultados estéticos sobre todo en los grupos anteriores, a base, en muchos casos, de desgastar tejido dentario existente. El objetivo de este artículo es presentar las carillas de porcelana como una de las técnicas reconstructivas adhesivas que proporcionan una estética dental excelente con una mínima alteración del tejido dentario.

Palabras clave: Carillas de porcelana, Restauración, Prótesis Adhesivas, Ácido fluorhídrico.

Abstract: Nowadays, aesthetics is an important part in social and professional relationships. The all-ceramic restorative systems have allowed us to improve our aesthetic results, mainly in the anterior region, at the expense in many cases- of removing existing tooth substance. The objective of this article is to present the porcelain veneers as one of the adhesive restorative techniques that provide excellent dental aesthetics with a minimum alteration of the tooth.

Key words: Porcelain veneers, Restoration, Adhesive restorations, Hydrofluoric acid.

BIBLID [1138-123X (2005)10:3; mayo-junio 241-368]

de Rábago-Vega J, Tello-Rodríguez Al. Carillas de porcelana como solución estética en dientes anteriores: informe de doce casos. RCOE 2005;10(3):273282 


\section{Introducción}

Actualmente vivimos en una sociedad cada vez más obsesionada con la apariencia estética, imponiéndose en muchas ocasiones este aspecto al puramente profesional. La cara es la primera parte del cuerpo que se ve cuando nos relacionamos; por tanto, la expresión facial es el aspecto más importante en la estética ya que cualquier defecto puede provocar el rechazo del observador o incluso, en muchas ocasiones, inseguridad o complejos en la persona que lo posee. Este es el motivo por el que debemos ofrecer a nuestros pacientes una atención especial en las técnicas estéticas que año tras año se vienen desarrollando ${ }^{1,2}$. El progreso de los composites, así como la evolución de las cerámicas, es un fiel exponente de las demandas de la población. De esta forma, las carillas de porcelana se han ido abriendo camino entre las diferentes técnicas reconstructivas de la sonrisa ${ }^{3}$.

\section{Evolución histórica}

La mayoría de los dentistas creen que los comienzos de la odontología cosmética se iniciaron con las resinas. Pero la sensación estética que proporciona la sonrisa se ha tenido en cuenta desde el primer retrato hasta el descubrimiento de la fotografía. Si observamos la mejora de la fotografía desde el siglo XIX hasta nuestros días, seremos conscientes del perfeccionamiento a que ha sido sometida. En el cine ocurrió lo mismo. En los años 20 mejoran las técnicas de proyección, apreciándose más los defectos estéticos. Por este motivo, los productores de Hollywood exigían a los actores una mayor perfección, especialmente en sus sonrisas, ya que no todos poseían una dentición perfecta. Por aquel entonces el Dr. Charles Pincus, dentista de Beverly Hills, intentaba mejorar el aspecto estético de sus pacientes, muchos de los cuales trabajaban en la industria cinematográfica. El reto era mejorar los primeros planos de las sonrisas con algo estético, cómodo, que no interfiriera con la función fonética y que se mantuviera en la boca el tiempo necesario durante el rodaje de las distintas secuencias cinematográficas. Desarrolló así las carillas de porcelana, que cumplían estos requisitos. La técnica consistía en cocer una capa muy fina de porcelana sobre papel de aluminio, diseñando de esta forma unas carillas ferulizadas que se pegaban temporalmente sobre los dientes del actor que iba a actuar".

El gran inconveniente de estas carillas era la falta de componentes de adhesión que posibilitara la estabilidad de estas reconstrucciones a largo plazo. En 1955, Buonocuore consigue grabar el esmalte dental, lo que supuso un paso importante en la adhesión al tejido dentario, pero no se conseguía adherir a las cerámicas ${ }^{5}$.

En 1972 el Dr. Alain Rochette publica un artículo donde describe un nuevo concepto de adhesión entre esmalte grabado y restauraciones de porcelana sin grabar. A ésta, la porcelana, se le aplicaba un producto, el silano, para facilitar la adhesión química de un cemento de resina sin partículas de relleno. Aunque los resultados obtenidos a lo largo de un año fueron excelentes, durante muchos años se dejó de hablar de su producto ${ }^{6}$. Pasaron los años, hasta que los doctores Simonsen y Calamia, en la década de los 80, descubren el efecto de grabado del ácido fluorhídrico sobre la cerámica. Es a partir de entonces cuando se puede decir que comienza el avance de las carillas de porcelana? ${ }^{7}$.

\section{Definición e indicaciones}

Las carillas de porcelana se pueden definir como una lámina relativamente fina de cerámica que se adhiere a la superficie vestibular de los dientes anteriores mediante resina compuesta y cuya única finalidad es la estética. Hoy en día se considera una de las técnicas de reconstrucción indirecta con resultados más favorables, tanto por su duración como por su aspecto estético (fig. 1 y 2). Dentro de las indicaciones podemos considerar las siguientes, cada una de ellas ilustrada con uno o más casos clínicos.

\section{Diastemas}

Los diastemas, y en particular el diastema medio interincisivo, son uno de los hallazgos más frecuentes en la práctica odontoestomatológica $a^{8^{*}}$ y pueden ser debidos a diversas causas: maloclusión, discrepancias en el tamaño dental o enfermedad periodontal (fig. 3A).

La incidencia de los diastemas varía enormemente con la edad y con la raza. Taylor describió, en 1939, que en los niños de hasta cinco años existía una incidencia de $97 \%$, cifra que disminuía a medida que los pacientes crecían. Lavelle, en un estudio realizado en el Reino Unido, determinó una mayor prevalencia de los diastemas 


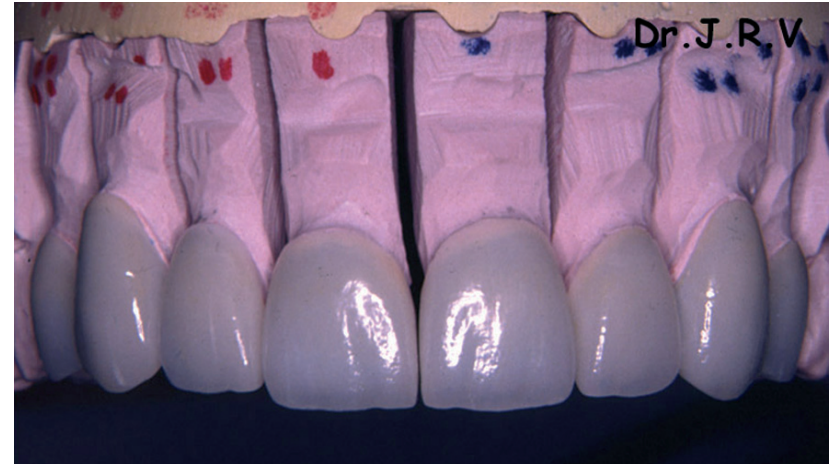

Figura 1. Carillas de porcelana.

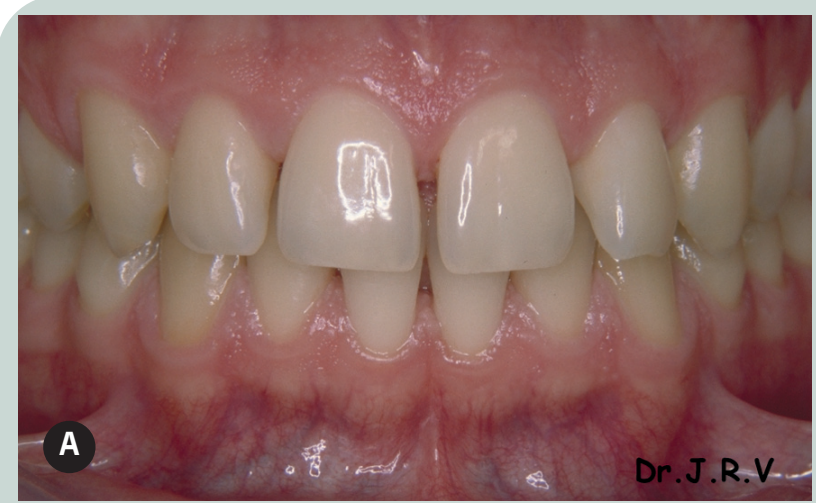

Figura 3A. Diastema. Antes.

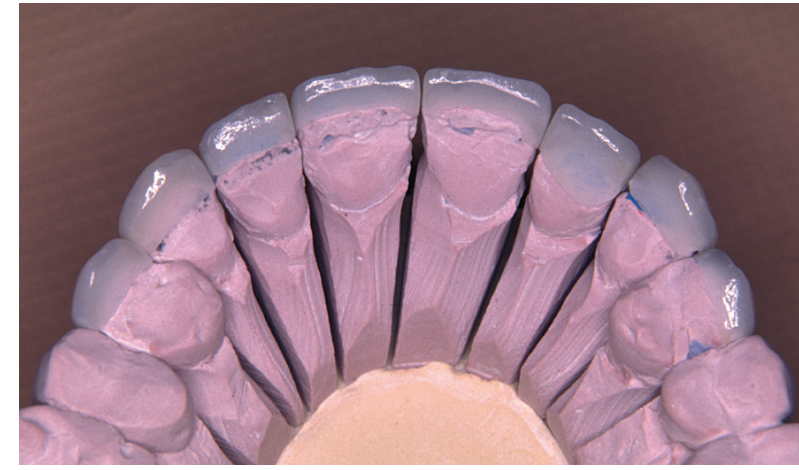

Figura 2. Carillas de porcelana.



Figura 3B. Diastema. Final.

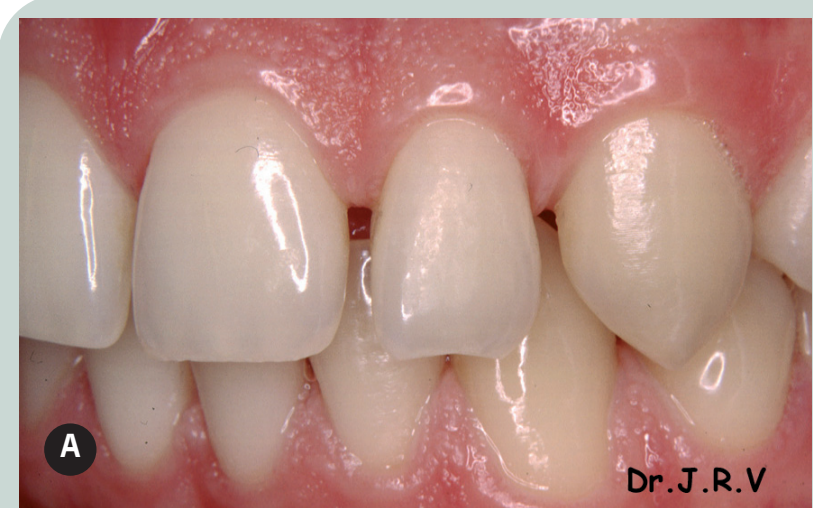

Figura 4A. Diastema vista lateral. Antes.

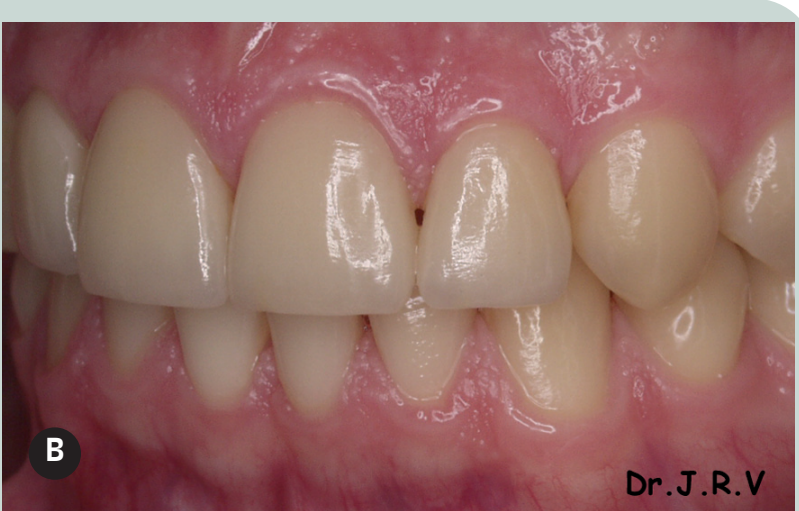

Figura 4B. Diastema vista lateral. Final. maxilares en la línea media en individuos de raza negra $(5,5 \%)$ que en los de raza blanca $(3,4 \%)$, así como en las personas de ascendencia asiática $(1,7 \%)$

Aunque es evidente que la prevalencia de los diastemas de la línea me- dia maxilar varía según la población y el grupo de edad, este fenómeno está asociado a un factor importante de maloclusión. En los niños en proceso de desarrollo, los diastemas de la línea media maxilar son normales. Sin embargo, en los adultos este efecto está asociado a la discrepancia en el tamaño de los dientes y a una excesiva superposición vertical de los incisivos. También puede originarse por la angulación mesio-distal de los incisivos, la inclinación labio-lingual de los incisivos, los frenillos y los procesos patoló- 


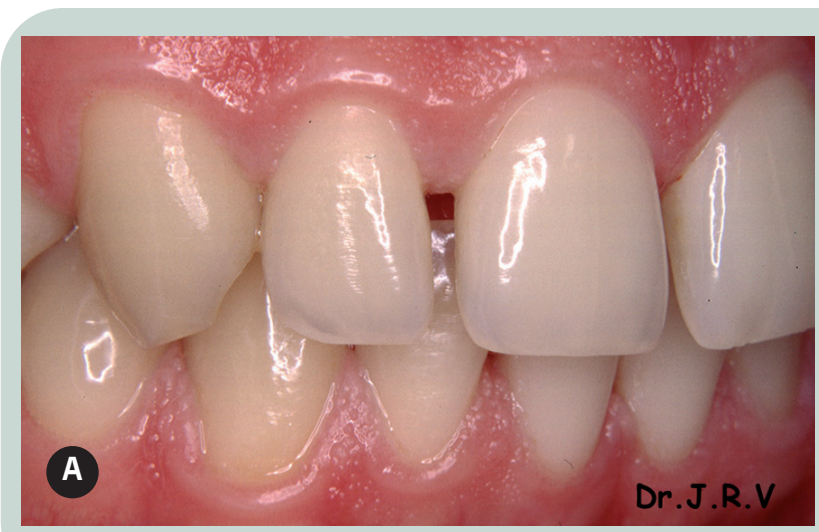

Figura 5A. Diastema vista lateral. Antes.

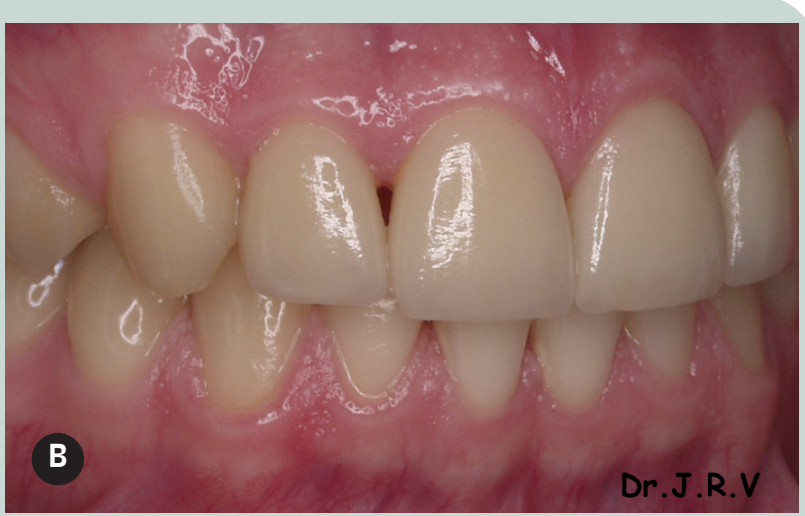

Figura 5B. Diastema vista lateral. Final.

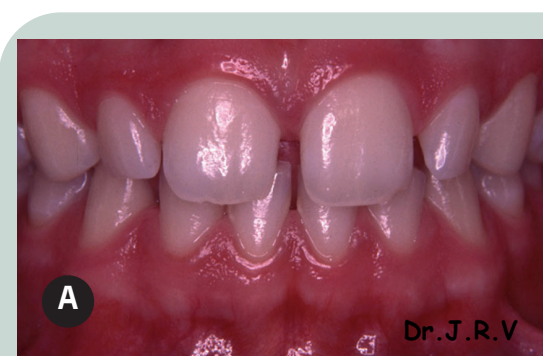

Figura 6A. Diastema vista frontal. Antes.

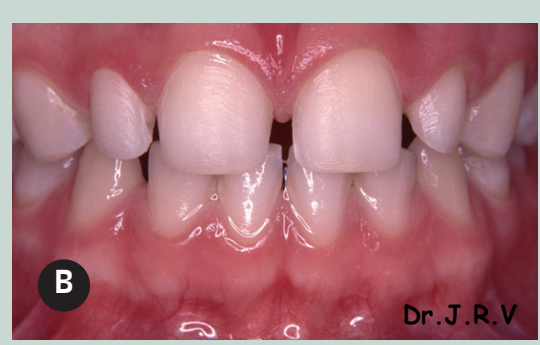

Figura 6B. Diastema. Tallado.

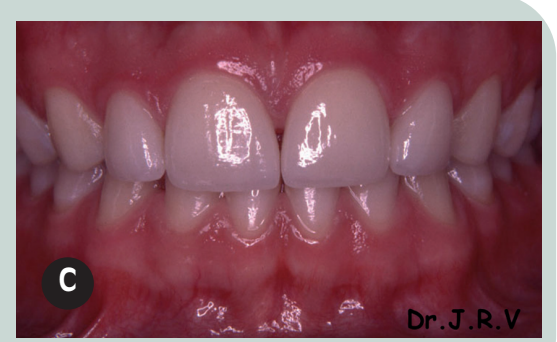

Figura 6C. Diastema. Final.

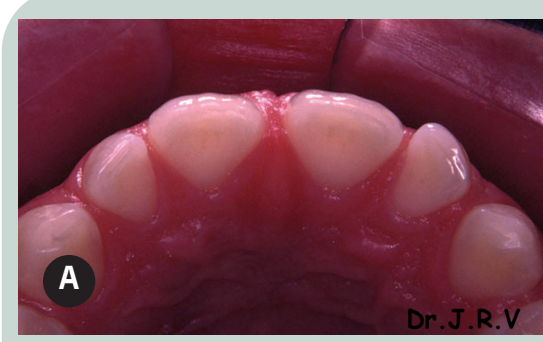

Figura 7A. Diastema vista oclusal. Antes.

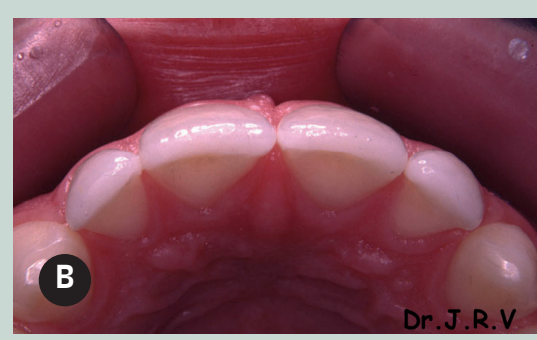

Figura 7B. Diastema vista oclusal. Final.

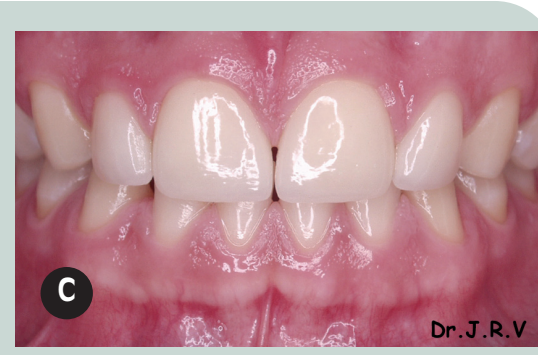

Figura 7C. Diastema. Final. Cuatro años después. gicos. La rehabilitación de casos de diastemas mediante carillas de porcelana en grupos anterosuperiores se considera una solución muy conservadora ${ }^{{ }^{*}}$, siempre y cuando el tratamiento ortodóncico no sea el indicado o el paciente no lo acepte (fig. 3 B, 4 A, B y 5 A, B).

Otra causa de diastemas es la discrepancia del tamaño de los dientes en relación a las arcadas de
Ios maxilares. La situación más frecuente es aquella en la que los incisivos centrales superiores adoptan una forma reducida en incisal y los laterales presentan una forma cónica, permitiendo la distalización de Ios incisivos centrales y provocando un diastema medial maxilar. Este problema se corregirá tan sólo con soluciones protésicas no ortodóncicas, adecuando la falta de espacio con las restauraciones (fig. 6 A, B, C y $7 \mathrm{~A}, \mathrm{~B}, \mathrm{C}$ ).

Existen otras causas de diastemas como la excesiva superposición vertical anterior, de cuya corrección se hace cargo el ortodoncista y que tan sólo se corregirá mediante prótesis cuando el paciente no acepte la ortodoncia como medio correctivo.

También los pacientes que padecen algún hábito, como la interposición lin- 


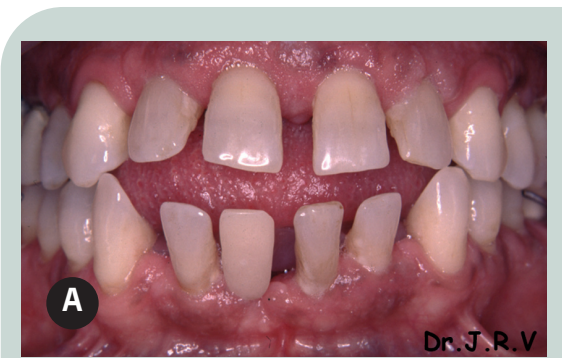

Figura 8 A. Interposición lingual. Antes.

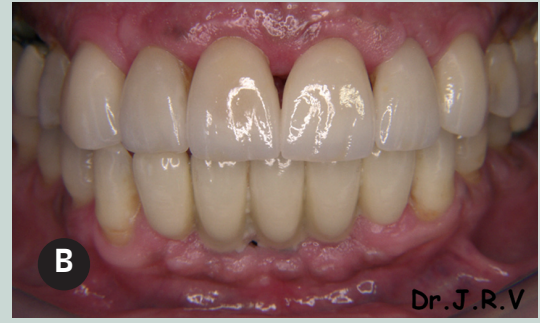

Figura 8B. Interposición lingual. Final.

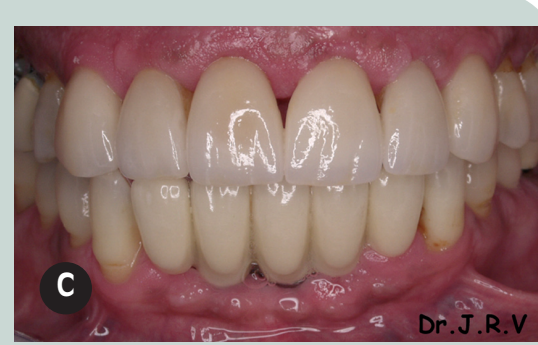

Figura 8C. Interposición lingual. Cinco años después.

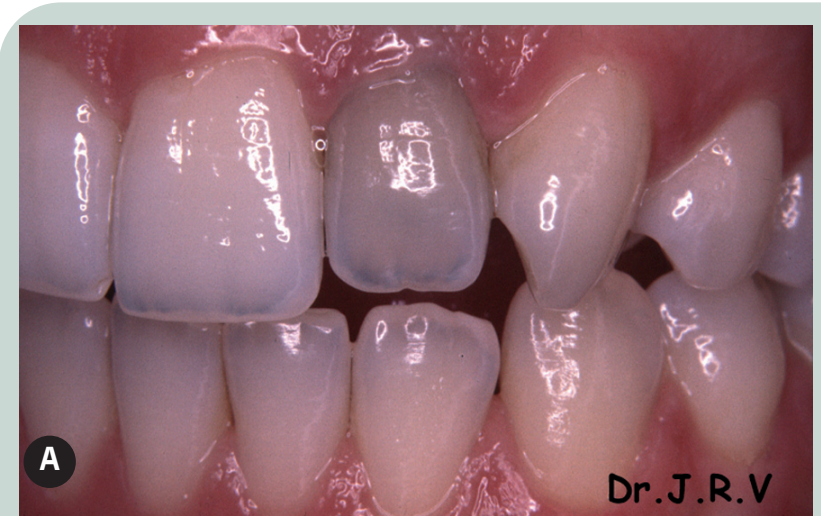

Figura 9A. Tinción endodóncica. Antes.

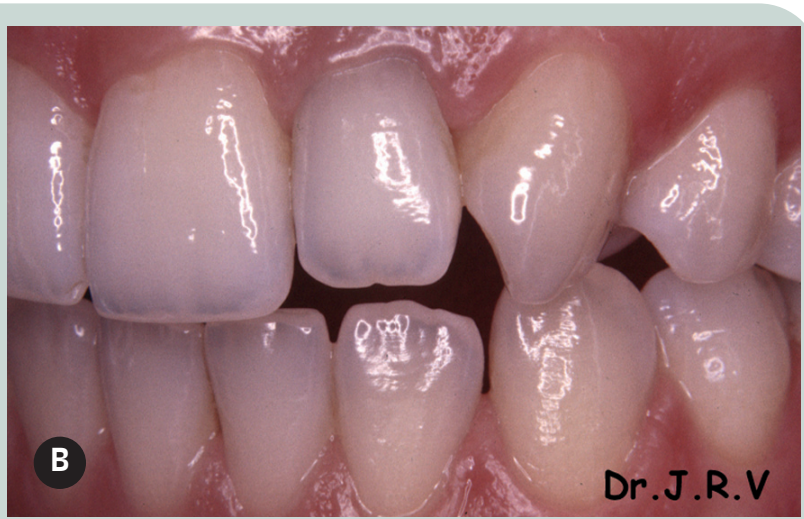

Figura 9B. Blanqueamiento. Después. gual asociada a enfermedad periodontal, pueden presentar diastemas. Estos pacientes muestran pérdida ósea generalizada con un notable aumento de su corona clínica. Ello determina un brazo de palanca excesivo, lo que provoca, en movimientos excéntricos (lateralidad y protrusión), una considerable movilidad produciendo la migración, generalmente en los grupos anterosuperiores. Para su rehabilitación, realizamos primero el tratamiento periodontal oportuno para, más tarde, cuando el periodonto y la mucosa oral se encuentren en perfecto estado de salud, terminar nuestra rehabilitación estética con la colocación de carillas de porcelana (fig. 8 A y B).

Como parte fundamental para el mantenimiento a largo plazo de estos casos, es necesaria la utilización de una férula o retenedor nocturno ya que la recuperación de estas alteraciones funcionales, sobre todo en pacientes adultos, resulta imposible (fig. $8 \mathrm{C}$ ).

\section{Tinciones dentarias}

Las tinciones dentarias pueden ser debidas a tres causas:

\section{a) Tratamientos endodóncicos}

Son las producidas por tratamientos endodóncicos en los que ha existido una hemorragia interna que con el tiempo acaba pigmentando el tejido dentario. En este caso somos partidarios de evitar en lo posible cualquier restauración protésica, debido a que con blanqueamientos internos se pueden conseguir resultados extraordinarios $^{10^{*}}$ (fig. 9 A y B).

\section{b) Tinciones dentarias medicamen- tosas}

Otro tipo de tinciones dentarias son las tinciones medicamentosas en pacientes tratados durante su infancia con tetraciclinas. Estos antibióticos, descubiertos en 1948, derivan químicamente de un núcleo tetracíclico -el octahidronastaceno carboxamida- que se caracteriza por ser intensamente fluorescente a la luz ultravioleta. Su efecto dañino en los dientes fue descubierto en $1956^{11}$.

Su administración durante el embarazo y en los niños que no han acabado la calcificación de la corona de los dientes definitivos, aproximadamente hasta los ocho años, produce un color amarillento durante la erupción para transformarse en marrón por la exposición a la luz ${ }^{12}$ 


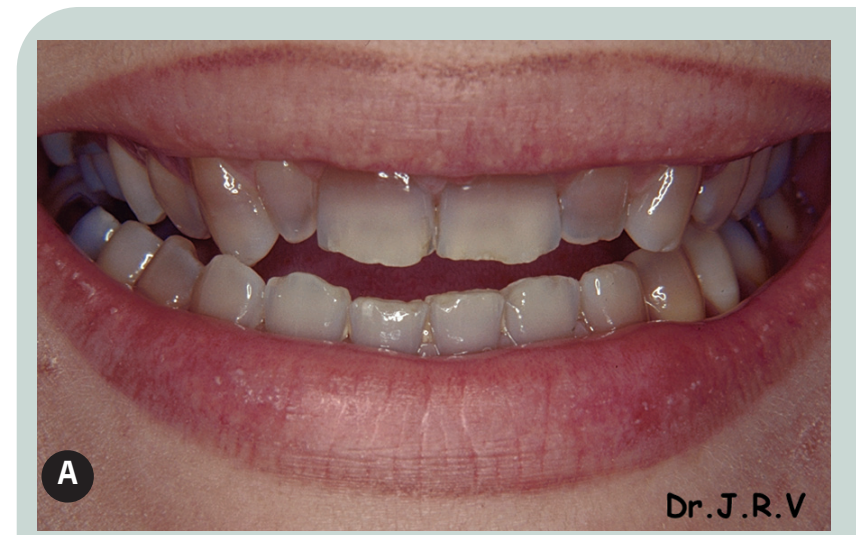

Figura 10A. Tinción por tetraciclinas. Antes.

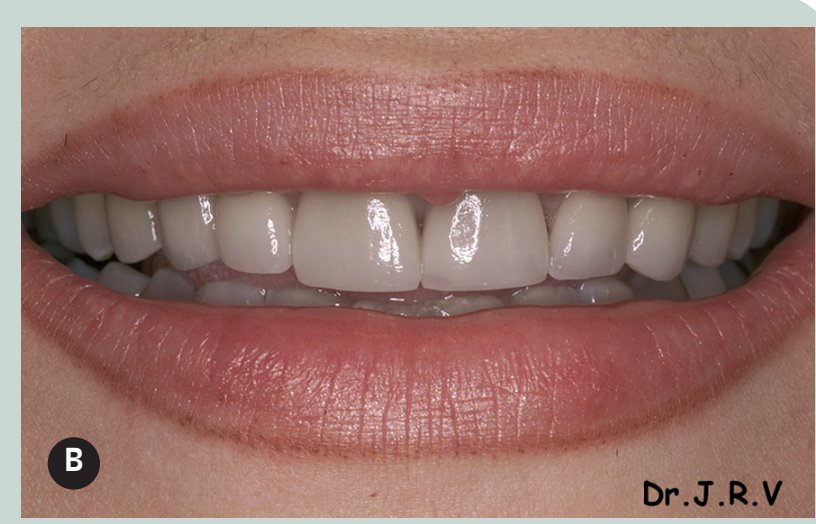

Figura 10B. Tinción por tetraciclinas. Final.

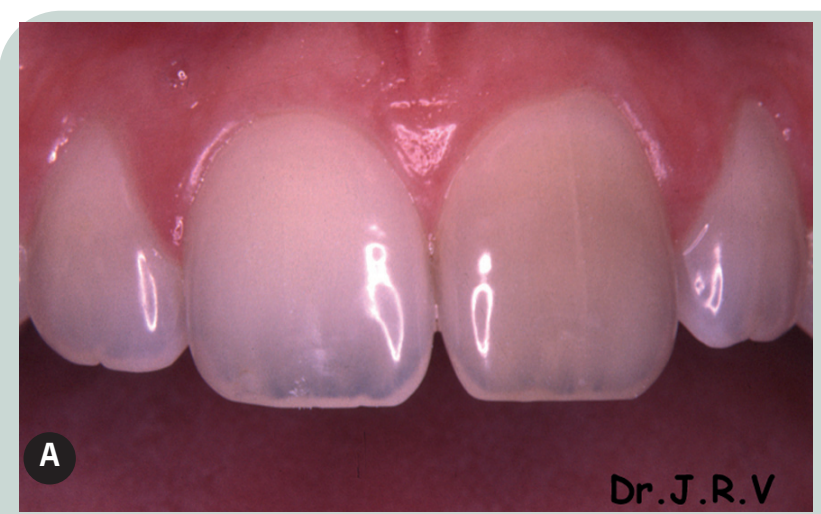

Figura 11A. Traumatismo. Antes.

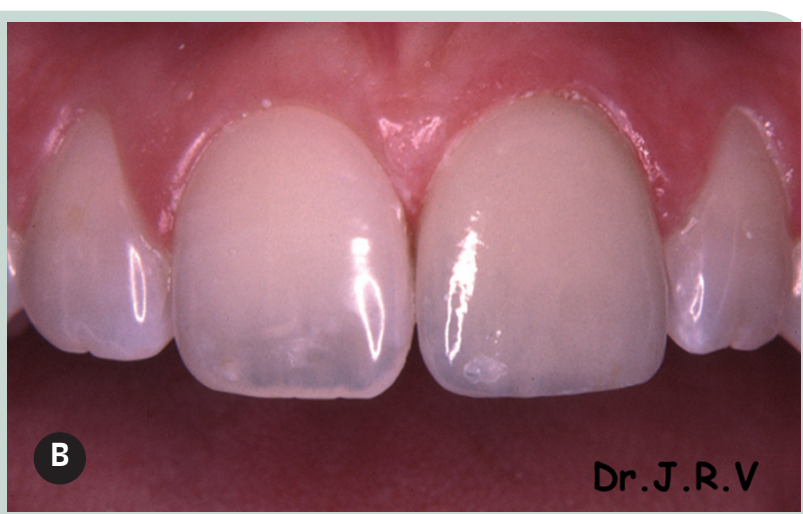

Figura 11B. Traumatismo. Final.
Estos casos pueden tratarse directamente con composites o con carillas de porcelana ${ }^{13^{*}}$. La elección de uno u otro dependerá de la habilidad del operador y de la dificultad de la restauración, aunque en muchas ocasiones $y$, debido a la aparición de diferentes opacificadores, las restauraciones directas con composites son una alternativa terapéutica que permite obtener resultados estéticos muy satisfactorios ${ }^{14}$.

En algunos casos la utilización combinada de técnicas ortodóncicas con restauraciones indirectas cerámicas resolverá favorablemente el caso a $\operatorname{tratar}^{15^{*}}$ (fig. $10 \mathrm{~A}$ y B)

\section{c) Tinciones dentarias debidas a traumatismos}

En algunos traumatismos se puede producir una hemorragia interna que ocasiona la descomposición local de la hemoglobina, penetrando pigmentos en el interior de los túbulos dentinarios y provocando a lo largo del tiempo una tinción dentaria importante. Clínicamente son dientes vitales y radiográficamente no presentan ninguna patología periapical, con lo cual está totalmente contraindicado un tratamiento endodóncico inmediato. En estos casos el blanqueamiento interno es imposible, siendo necesario colocar carillas de cerámica, composites o coronas de recubrimiento total (fig. 11 A y B).

\section{Dientes conoides}

Los dientes conoides generalmente afectan a los incisivos laterales superiores y pueden ser unilaterales o bilaterales. Esta situación es muy favorable para las carillas de cerámica ya que la preparación es prácticamente nula y la agresión al tejido dentario mínima, por lo que se considera una muy buena solución terapéutica, que armoniza el tamaño dentario y estabiliza la oclu$\operatorname{sión}^{16}$ (fig. 12 A, B).

En este tipo de malformación dentaria, las carillas de porcelana han de ser muy amplias para cerrar los espacios interproximales. De esta forma, en muchos casos vienen a te- 


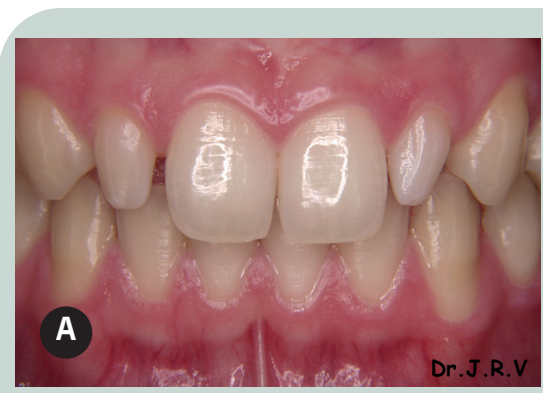

Figura 12A. Dientes conoides bilaterales. Antes.

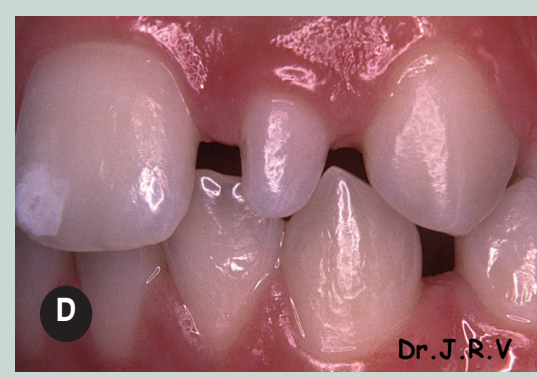

Figura 12D. Dientes conoides bilaterales. Antes.

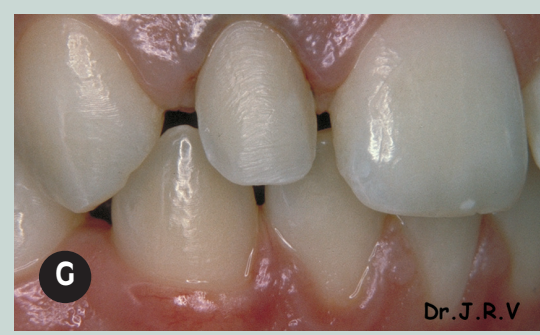

Figura 12G. Dientes conoides bilaterales. Tallado.

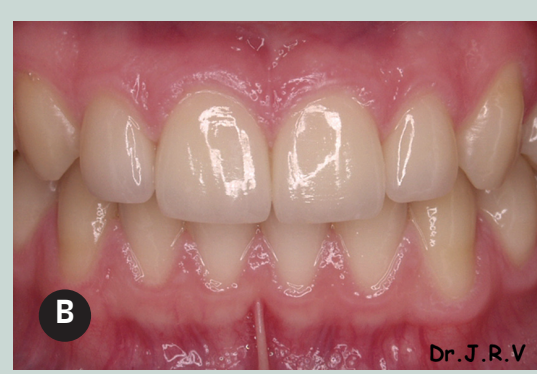

Figura 12B. Dientes conoides bilaterales. Antes.

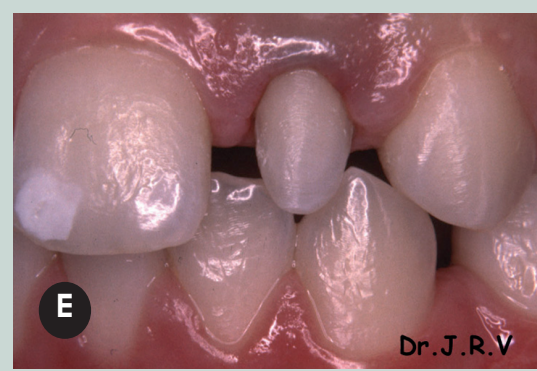

Figura 12E. Dientes conoides bilaterales. Tallado.

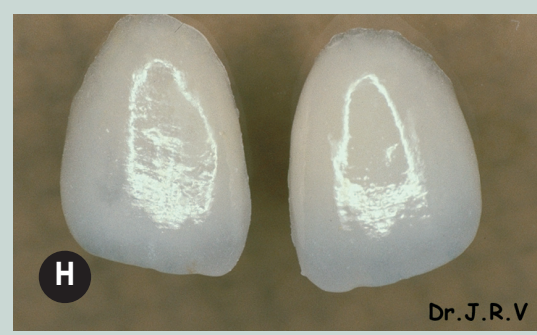

Figura 12H. Dientes conoides bilaterales. Carillas.

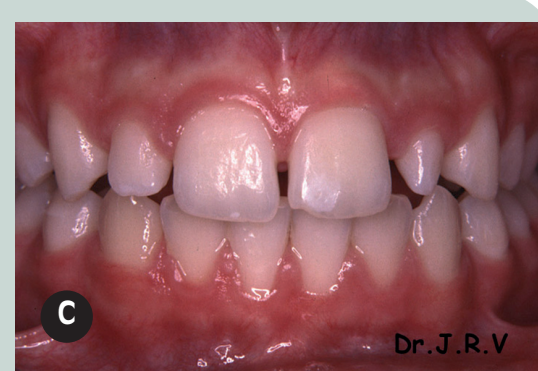

Figura 12C. Dientes conoides bilaterales. Antes.

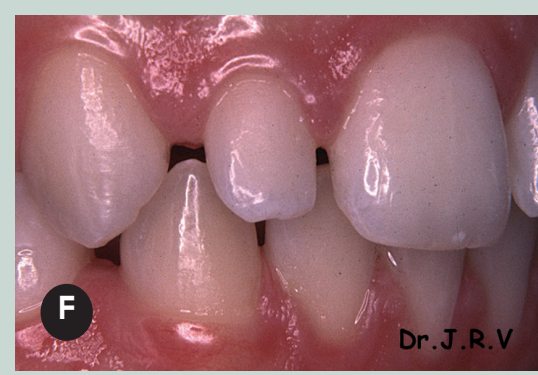

Figura 12F. Dientes conoides bilaterales. Antes.

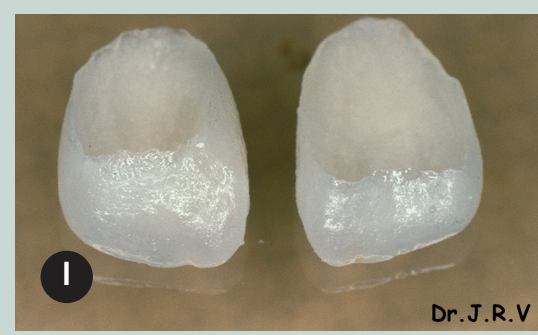

Figura 12I. Dientes conoides bilaterales. Carillas. ner la forma casi de una corona de recubrimiento total con una terminación cervical en filo de cuchillo (fig. 12 C,D,E, F, G, H e I.

También es frecuente la asociación de un diente lateral conoide con la agenesia del contralateral. En estos casos colocaremos en los dientes conoides una carilla de cerámica y en las agenesias de laterales un implante osteointegrado y una corona unitaria (fig. $13 \mathrm{~A}, \mathrm{~B}$ y C).
Malposiciones moderadas

La ortodoncia es la principal modalidad terapéutica de las malposiciones dentarias. Pero la colocación de la aparatología fija coincide, en muchas ocasiones, con pacientes en edades puberales "difíciles» que no aceptan este tipo de tratamiento, lo cual limita mucho su aplicación.

Las carillas de porcelana son tratamientos restauradores más rápidos que la ortodoncia y disimulan ligeras asimetrías y malposicione ${ }^{17}$ y, aunque mucho más agresivos, se admiten ya que se rechaza la ortodoncia.

Por supuesto que estos casos tratados con carillas de cerámica han de ser siempre malposiciones leves. Para las malposiciones exageradas, la única solución será la rehabilitadora, bien con tratamiento ortodóncico o con coronas de recubrimiento total (fig. 14 A, B, C y D). 


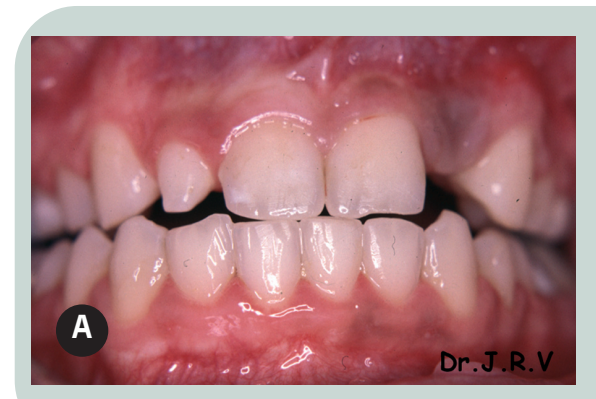

Figura 13A. Agenesias. Antes.

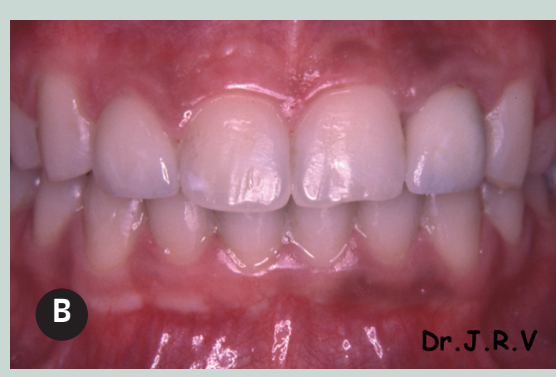

Figura 13B. Agenesias. Final.

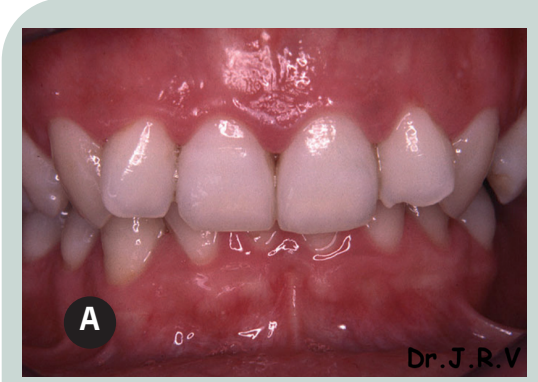

Figura 14A. Malposición. Antes.

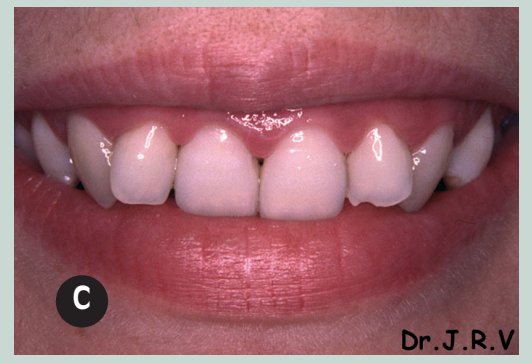

Figura 14C. Malposición. Antes.

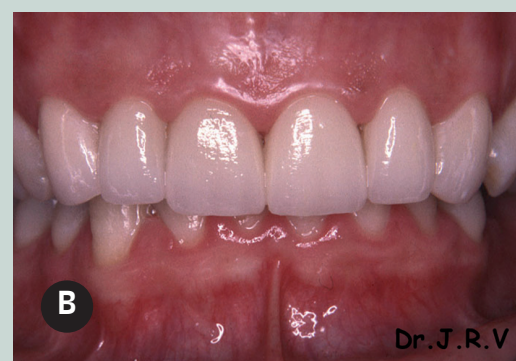

Figura 14B. Malposición. Final.

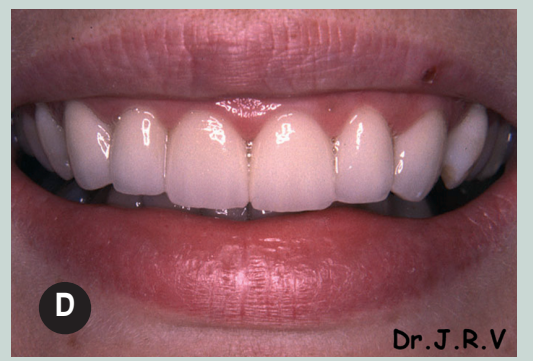

Figura 14D. Malposición. Final.

\section{Dientes deciduos}

Esta es una situación poco usual en nuestros consultorios. La presentamos como indicación de carillas de cerámica en aquellos casos en los que el paciente presenta dientes deciduos y por causas estéticas desea rehabilitar estos dientes. El caso más frecuente son los caninos superiores donde una malposición de los definitivos o una erupción atípica de éstos impiden su proyección ideal, haciéndolos permanecer incluidos en el paladar. Esta situación hace que los caninos deciduos permanezcan en la boca, pero al ser

más pequeños y de un color diferente al de los dientes adyacentes definitivos provocan una desarmonía en la estética general (fig. 15 A y B). La carilla de porcelana colocada en un canino superior deciduo permite recontornear incisal e interproximalmente el diente, preservando la estructura dentaria y salvaguardando la vitalidad de dicho canino ${ }^{18^{\star}}$. Será necesario que la zona radicular no haya sufrido reabsorción y que la oclusión final sea función de grupo (fig. 15 C y D).

Una de las grandes dudas está en la calidad del esmalte en los dientes

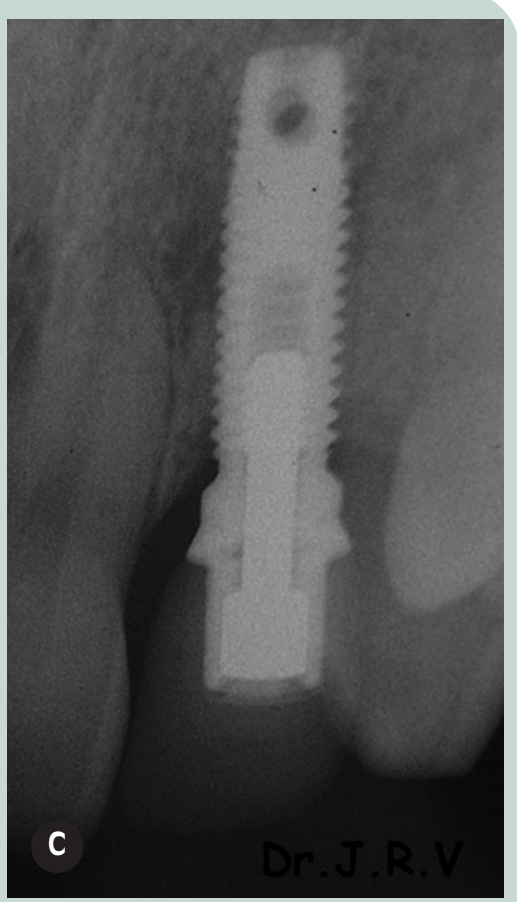

Figura 13C. Agenesias. RX Intaoral.

deciduos que limitarían la adhesión de las carillas y su prematuro fracaso. En el estudio de Da Silva y cols se contrastan las características ultraestructurales de los tejidos duros del diente definitivo con las de la dentición temporal. La conclusión fue que siendo las estructuras similares, el esmalte deciduo presenta la denominada línea neonatal de defectos en la zona cervical y de una capa superficial de esmalte aprismático. Por consiguiente, los tallados deberán ser selectivos, preservando la gran mayoría de tejido de esmalte ${ }^{19^{* \star}}$.

\section{Fracturas dentarias}

La reconstrucción con composite de las fracturas dentarias leves es una solución muy favorable debido a los avances en las técnicas de adhesión ${ }^{20}$ (fig. $16 \mathrm{~A}$ y B). 


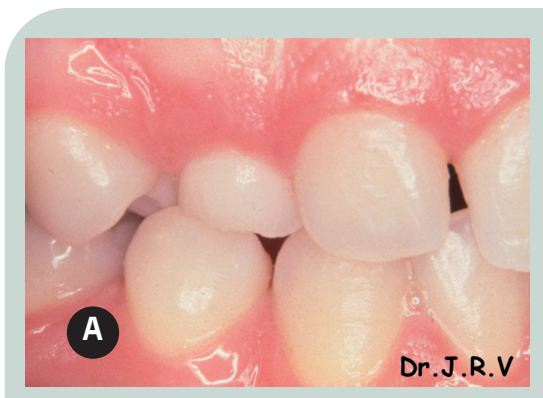

Figura 15A. Dientes deciduos. Antes.

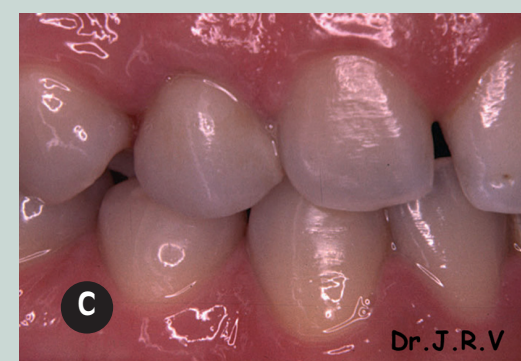

Figura 15C. Dientes deciduos. Final.

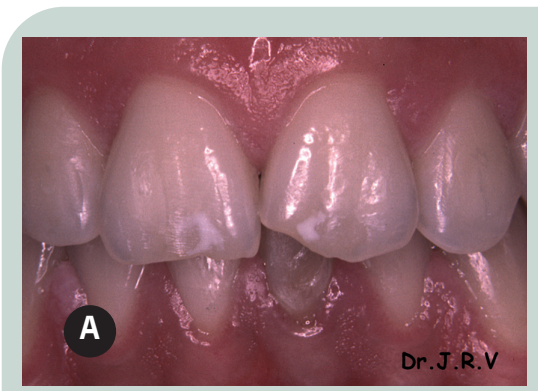

Figura 16A. Fractura. Antes.

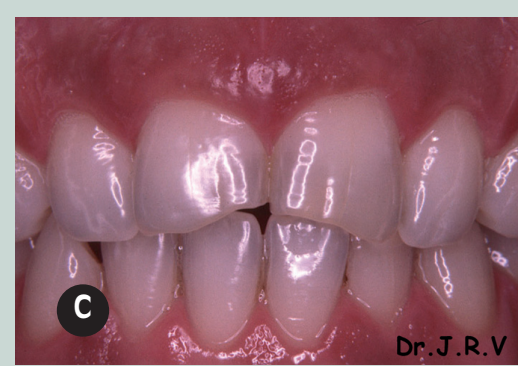

Figura 16C. Fractura. Antes.

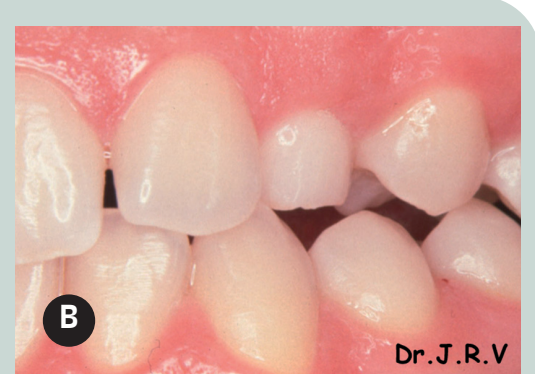

Figura 15B. Dientes deciduos. Antes.

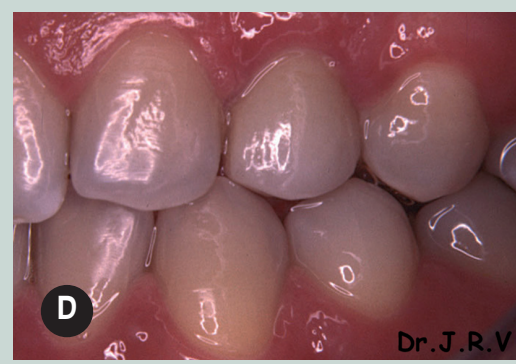

Figura 15D. Dientes deciduos. Final.

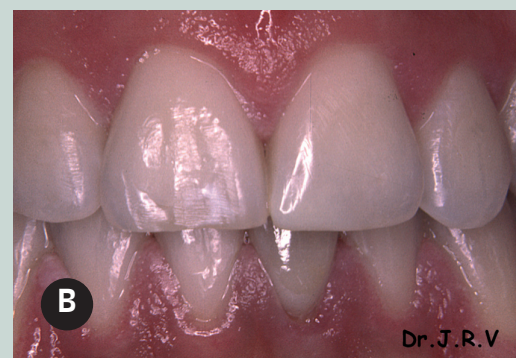

Figura 16B. Reconstrucción con composite

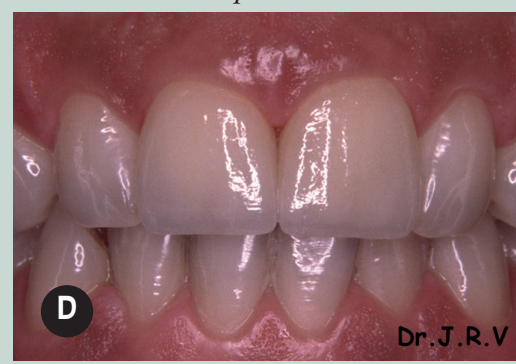

Figura 16D. Fractura. Final. Carillas de porcelana.

En los casos de fracturas de bordes incisales demasiado amplias, en las que la reconstrucción con materiales com- puestos esté comprometida, su rehabilitación con carillas de cerámica será más resistente, ya que la combinación de composite y cerámica para restablecer un diente fracturado puede reproducir el comportamiento biomecánico del diente intacto ${ }^{21}$ (fig. 16 C y D).

\section{Conclusiones}

1. Las carillas de porcelana son una alternativa estética a tener en cuenta por sus resultados clínicos a largo plazo y por la agresión mínima al tejido dentario.

2. No todos los casos se deben tratar con esta técnica, ya que existen otras alternativas como los composites que, bien manejados, pueden suplirlas, obteniendo unos resultados estéticos muy favorables.

3. Los casos de malposiciones dentarias y diastemas se deben tratar con ortodoncia y sólo se utilizarán las carillas de porcelana cuando el paciente no acepte este tratamiento.

4. Las tinciones dentarias se tratarán con blanqueadores. Si fueran muy intensas, con carillas. Si afecta a un incisivo central superior, la mejor alternativa será el composite ya que se podrá conseguir un mejor acercamiento del color al del diente contralateral.

5. Una indicación absoluta de las carillas de porcelana serán los dientes conoides, por su poca preparación y excelentes resultados.

6. Los dientes deciduos se pueden rehabilitar con carillas siempre que se preserve la estructura de esmalte necesaria para obtener una buena adhesión.

7. Las fracturas dentarias leves se rehabilitarán con composites. Si afectan al tercio incisal, se dispondrá de la opción de carillas. 


\section{Bibliografia recomendada}

Para profundizar en la lectura de este tema, el/los autor/es considera/an interesantes los artículos que aparecen señalados del siguiente modo: *de interés ${ }^{*}$ de especial interés.

1. Becerra Santos, G. Fundamentos estéticos en rehabilitación oral. Parte I: Factores que influyen en la estética dental. Proporciones «doradas». Estética facial. Rev Int Prótesis Estomatol 2001;3(4):247-52.

2. Becerra Santos, G. Fundamentos estéticos en rehabilitación oral. Parte II: Tipos de sonrisa. Consideraciones acerca del color. Percepciones visuales e ilusiones ópticas. Rev Int Prótesis Estomatol 2001;3(5):343-51.

3. Christensen GJ. The state of the art in esthetic restorative dentistry. $\mathrm{J}$ Am Dent Assoc 1997;128(9):1315-7.

4. Pincus CL. Building mouth personality. J Calif Dent Ass 1938;14(4):125-9.

5. Buonocuore MA. A simple method of increasing the adhesion of acrylic fillings to ename surfaces. J Dent Res 1955;34:849-53.

6. Rochette AL. A ceramic restoration bonded by etched enamel and resin for fractured incisors. J Prosthet Dent 1975;33(3):287-93.

7. Calamia JR. Etched porcelain facial veneers: a new treatment modality based on scientific and clinical evidence. NYJ Dent 1983;53:255-9.

8*. De Carlos-Villafranca F, Cueto-Suárez M, Cobo-Plana-JM, Fernández-Mondragón, MP. Los diastemas, etiología y soluciones: [1] etiología. RCOE 2000;5(4):363-71.

El autor relata con bastante detalle todas las posibles causas de diastemas, deteniéndose en cada una de ellas.
9*. Pensler AV. Multiple-diastema porcelain laminate veneers: a case study. Compendium 1993;14(11):1470, 1472, 1476-7.

En este artículo se muestra una preparación muy conservadora de seis dientes anterosuperiores para colocar carillas de porcelana.

10*. Attin T, Paque F, Ajam F, Lennon AM. Review of the current status of tooth whitening with the walking bleach technique. Int Endod J 2003;36(5):313-29.

Se describe el procedimiento de blanqueamiento interno y se contraindica el uso del calor

11. Schwachman $H$. The tetracyclines applied pharmacology. Pediatr Clin N Amer 1956;3:295-303.

12. Urist MR. Visen HK. Chemical reactivity of mineralized tissue with oxitetracycline. Arch Pathol 1963;76:484

13*. Garber D. Porcelain laminate veneers: ten years later. Part 1: Tooth preparation. J Esthet Dent 1993;5:56-62.

Resumen de casos de carillas a diez años.

14. Lui JL. Treatment of discoloured anterior teeth using a simple veneering technique. Dent J Malays 1987;9(2):19-22

15*. Cooke MS, Wei SH. Esthetic treatment of severe tetracycline staining with orthodontics and veneers: a case report. Quintessence In 1994;25(3):161-5.

Se expone el tratamiento combinado de ortodoncia y carillas en un caso tinción por tetraciclinas.
16. Miyajima K, Shirakawa K, Senda A. Application of porcelain veneers following orthodontic treatment. J Can Dent Assoc 1993; 59(2):167-70.

17. Tipton PA. Aesthetic tooth alignment using etched porcelain restorations. Pract Periodontics Aesthet Dent 2001;13(7):551-5

18*. Aristidis GA. Etched porcelain veneer restoration of a primary tooth: a clinical report. J Prosthet Dent 2000;83(5):504-7.

Se presenta el caso de un canino deciduo que se mantuvo en un adulto y se reconstruyó con una carilla de porcelana extendida a la cara palatina y que permitió aumentar la longitud del diente.

19**. Da Silva L, Fraga JM, Santana U, Guitian F. UItraestructura de los tejidos duros del diente. Comparación de dientes definitivos y temporales. Arch Odontoestomatol 1994;10(1):16-28. Se detalla la diferencia que existe entre las estructuras de los tejidos duros del diente permanente y el temporal.

20. Andreasen FM, Daugaard-Jensen J, Munksgaard EC. Reinforcement of bonded crown fractured incisors with porcelain veneers. Endod Dent Traumatol 1991;7(2):78-83.

21. Magne P, Douglas WH. Optimization of resilience and stress distribution in porcelain veneers for the treatment of crown-fractured incisors. Int $\mathrm{J}$ Periodontics Restorative Dent 1999;19(6):543-53. 